\title{
Creating Site-Specific Training Tools: No Longer Wishful Thinking
}

Steven B. Barlow

EM Facility/Biology, San Diego State University, 5500 Campanile Dr., San Diego CA 92182-4614

Teaching and training users how to operate laboratory equipment is a time-intensive practice. Many new users are students, who have generally high turnover rates; after they complete a class project or degree program, they leave the facility. In addition, users often divide their time among a variety of techniques and equipment. With episodic usage, researchers tend to forget details of instrument operation and need to be retrained. Academic core facilities must balance their productivity against the time it takes to train and retrain an ever-changing parade of users.

Books, videos, CDs, and websites that highlight the use and theory of operation of equipment already exist. However, these resources provide only general information; they are not applicable to training users in the operational procedures and maintenance guidelines for particular instruments in your laboratory. Site-specific multimedia teaching and training resources, such as films, CDs, videos, or DVDs, if readily available, would enable users to refresh and retrain themselves.

In the past, producing training videos or equivalent teaching aides required expensive resources: camera, crew, editing equipment, and the like. However, there are now several low-cost, easy-touse programs for digitizing and editing video images, inserting them into a sequence along with title boards and still images, then exporting this edited session to a CD, DVD, or video tape. The Electron Microscope Facility at San Diego State University is using video editing software to create training CDs for in-house equipment and has thereby reduced the required training time by half. Using these CDs ensures a standardized presentation so that each user receives uniform instruction. Users can review the CDs as often as necessary to learn a procedure; for users whose native language is not English, this may be especially useful. The CDs (DVDs in the future) are inexpensive to duplicate and readily viewable on or off site at the researcher's convenience. Users can self-conduct a refresher session simply by reviewing a CD.

The production methodology for creating training CDs in the SDSU EM Facility is outlined below and will be demonstrated at this tutorial. The approach is similar to that of presenting a laboratory demonstration.

1) Develop a storyline and production scenario--Planning reduces the need for subsequent manipulation. If you have been training users for any length of time, you have already created your script: it is your well-honed presentation of the standardized protocol for a given piece of equipment. Before recording the tutorial, be sure the camera operator understands how to record the various scenes of the demonstration (e.g., changing shots or zooming too rapidly can be disorienting to the viewer). Make sure that there is sufficient lighting and that you will not be blocking the lighting, the camera, or the equipment during the recording. Remember that however you appear will be seen for years to come; so dress appropriately and comb your hair! Try a short practice tutorial and critically review it before embarking on the final shoot. Although this may sound daunting, in practice, after producing your first tutorial, the rest come easily. Spending time practicing before the shoot will save a great deal of time in the editing phase. 
2) Record the tutorial--We use a tripod mounted digital video camera. Any video camera is appropriate, however, even one of the laboratory cameras that has no sound input. (You can always add a soundtrack during editing.) Before starting to record, turn off pagers and telephones to avoid the recording of rogue sounds. If the presenter misspeaks during the shoot, redo that part of the scene immediately. It is easier to redo a part of a scene while equipment and supplies are in place than it is to edit a mistake later, or to plan to reshoot on a different day. Set up the camera and record all sequences for a given camera orientation before moving to a different location, even if it means that your protocol is filmed out of sequence. You will rearrange the sequences in correct order during the editing. Include short pauses in the narration - these pauses make it easier to insert, remove, or replace video clips or text during the editing.

3) Digitize the recording--The tutorial, once recorded, needs to be transferred to the computer. Digital video (DV) cameras and newer Macintosh computers are configured to exchange video data via Firewire and the software program iMovie. Plug the DV camera into the Mac using Firewire, open iMovie, and the video clip playing on the camera is automatically transferred to the computer. A Wintel machine running Windows XP requires an optional Firewire card to exchange digital video clips to the software program Movie Maker. To exchange video from an analog video camera or VCR, you need a video capture board and driver, such as the ATI All-In-Wonder card. You can transfer between analog and digital sources with a card like this.

4) Edit the recording--The latest computer operating systems contain simple video editing software: iMovie for the Mac running OS 9.1 or higher and Windows Movie Maker for Wintel machines running Windows XP. Video editing programs generally open a video viewing window with side and bottom editing areas. Video clips or still images from your camera or VCR are stored to the right of the viewer as slides. You can then edit each clip for content in the viewing window and drag it to its proper position in the video timeline below. You can modify soundtracks, if necessary, as part of the video clip editing. You can create or select new text slides and various effects to create transitions between segments. The final tutorial is assembled step by step in via a video timeline function. One hour of uncompressed video fills about $13 \mathrm{GBs}$ of memory, so a lot of disk space and plenty of RAM are an absolute necessity.

5) Store the tutorial--Save your movie by exporting it in DVD, videotape, CD, or Web-compatible format. DVD or videotape provides the best image quality; the others suffer various degrees of degradation of resolution. The CD-ROM format, which gives an acceptable intermediate resolution, is standard on most computers and is cheap and easy to copy and distribute. As DVD players become standard accessories, the high quality DVD format will be the best choice for recording and distributing tutorials. If you choose to record your tutorial to videotape, you will have a high quality image but users will need a VCR and monitor to view the tape. The tutorial can also be mounted on the Web for ready access by users and students (e.g., http://www.sci.sdsu.edu/emfacility).

In conclusion, easy to use digital video editors make it possible to create inexpensive, site-specific training tools, as well as class-specific teaching aids. The considerable savings of training time on the part of the facility staff is well worth the initial investment of production time. 